\title{
64907 "Green" Biopolymers for Improved Decontamination of Metals from Surfaces: Sorptive Characterization and Coating Properties
}

\author{
Publication Date: June 15, 2001
}

Lead Principal Investigator: Dr. Brian H. Davison, Oak Ridge National Laboratory, PO Box 2008, MS 6226, Oak Ridge, TN 37831, (865) 576-8522; davisonbh@ornl.gov

Co-Investigator: Dr. Tanya Kuritz, Oak Ridge National Laboratory, (865) 241-6013; ztk@ ornl.gov

\section{RESEARCH OBJECTIVE}

The proposed research aims to develop a fundamental understanding of important biological and physical chemical parameters for effective decontamination of metal surfaces using environmentally benign aqueous-based biopolymer solutions. Understanding how heavy metal-chelating biopolymers coat and interact with contaminated surfaces will benefit the development of novel, safe, easy-to-apply decontamination methodologies for removal of radionuclides and heavy metals. The benefits of these methodologies will include the following: decreased exposure hazards for workers; decreased secondary waste generation; increased efficiency of decontamination; positive public appeal and development of novel, nature-friendly business opportunities; and lower cost of cleanup to the government.

We propose to use aqueous biopolymer solutions to coat a contaminated metal surface (i.e., steel), solubilize the heavy metals (e.g., uranium) from the surface, and bind the heavy metals into the biopolymer. The biopolymer coating (containing the immobilized hazardous metal contaminants) will then be removed as a viscous film, as a dry powder, or by washing. This "apply, wait, and remove" procedure will reduce the amount of worker time spent in decontamination activities.

\section{RESEARCH PROGRESS AND IMPLICATIONS}

We are over 2 years of 3 years into research on "green" biopolymers for a decontamination system that is efficient, safe, and environmentally friendly. We have successfully confirmed the core hypothesis of that proposal: to use aqueous biopolymer solutions to coat a contaminated metal surface (i.e., steel), solubilize the heavy metals (uranium) from the surface, bind the heavy metals into the biopolymer, and remove the biopolymer-radionuclide complex.

Highlight. A biopolymer system was developed in our research, that, at present, removes $>80 \%$ of the uranium (VI) from contaminated steel coupons. These results required us to screen and separate the appropriate fraction of several promising biopolymers, develop a technique to quantitatively construct contaminated coupons, and then apply and remove coats of contaminated biopolymer (Davison and Kuritz, 2000).

Biopolymer testing. We have measured and confirmed the metal sorptive capabilities of several biopolymers at radionuclide concentrations of up to $0.2 \mathrm{~g} \mathrm{U} / \mathrm{g}$ biomass and have selected a promising cyanobacterium, Nostoc muscorum strain HPDP22 (gift of L. Sirenko, Institute of Hydrobiology, Academy of Sciences, Ukraine). We have also isolated and analyzed the effectiveness of metal-binding and rheological properties of whole biomass and of different biopolymer fractions (different types of biopolymers from the same microorganism) from several sheath-producing algal species. As a result, we have selected the fraction that is most promising for metal chelation on surfaces from Nostoc-produced biopolymers. This charged soluble fraction (RPS) had a viscosity just under $20 \mathrm{cp}$.

Different microalgal biomasses and biopolymer fractions showed widely varying sorption properties with regard to barium, cadmium, iron, copper, and uranium as measured by inductively-coupled plasma emission spectroscopy (ICP). Comparative isotherms for uranium (VI) and cadmium shown in Figure 1 indicate the greater specificity and capacity for uranyl by the Nostoc sp. High uranyl capacity and specificity is desired to allow for competition with other metal ions that may be present. The capacity greater than $0.2 \mathrm{~g} / \mathrm{g}$ is equivalent to ion exchange resins. 
Biopolymer production. Algal cultures were grown in photobioreactors for 10-12 days in mineral media, then left to mature for an additional 10-12 days. "Maturation" of cyanobacteria is a poorly understood process in which these microorganisms produce copious quantities of biopolymers, predominantly polysaccharides. In our experiments, we tested the following biopolymers under different application conditions: (1) dry; whole-fraction biomass, (2) the insoluble polysaccharide fraction (EPS); and (3) the soluble polysaccharide fraction (RPS). We examined polymer abundance, process cost, and rheological properties of the fractions, and selected the RPS fraction of biopolymers produced by Nostoc muscorum HPDP22 for all second- and third-year work. This organism grows easily on minerals in the presence of light and produces large amounts of chelating biopolymers upon "maturation".

Development of quantified contaminated sample coupons. A critical ancillary achievement of the project has been the development and validation of a method to quantitatively apply and "fix" uranyl contamination onto a metal surface (steel) and measure the resulting alpha radiation; this was essential to quantify contaminant removal. Coupon preparation was aided by the prior work of Pierce (1960) and Demmer (1994) for development of simulated contaminated surfaces. To prepare a 'hot' coupon, a silicone epoxy was used to create a dike at the coupon edge; this allowed a uniform layer of contaminated liquid to remain on the surface; the coupon was then oven-baked $\left(100^{\circ} \mathrm{C}\right)$ until the liquid evaporated (Figure 2). A simple, quantifiable rinse was then used to remove "easily removed" contamination ( $<5 \%)$; a subsequent aggressive smear only removed $2 \%$ more. This was presumed to be the "loose" or transferable contamination, with the remainder presumed to be "fixed." Several methods were used to quantify contaminant surface concentrations; we primarily rely on direct alpha disintegration counts using a sample counter. Multiple readings on single coupons varied about $1 \%$; coupon to coupon variability was $5 \%$. The reproducible use of this instrument required construction of the coupons as $3.8-\mathrm{cm}$ nonpolished A36 carbon steel discs.

Tests of biopolymer decontamination of contaminated steel coupons. An example of a representative experiment follows. Two polymer fractions (RPS and EPS) were isolated from mature N. muscorum HPDP22. by centrifugation. The cell pellet was used for the isolation of another, cell-bound, insoluble biopolymer fraction (EPS). The two formulations (and a whole dried biomass control) were applied individually to steel coupons and allowed to dry.

The whole biomass and the insoluble biopolymer fraction were more difficult to remove from the surface after treatment than the soluble fraction; the latter of these formed easily-removable flakes. In a procedure similar to those performed on ion-exchange resins, the soluble biopolymer was pre-treated in a low ionic strength buffer to render it uncharged (near neutral $\mathrm{pH}$ ) or was positively charged by adjusting to $\mathrm{pH}$ 3. Figure 3 shows results from six individually tracked contaminated coupons - in each case, the biopolymer was applied as a coat, dried, and then removed from the coupons. Comparing the "before" and "after" alpha counts, the biopolymer successfully removed much of the radioactive contamination (Davison, et al., 1999) after a single application. After a second application of the charged biopolymer, over $80 \%$ of the contamination was removed. In our experimental estimates, $0.02 \mathrm{~mL}$ containing $5 \mathrm{mg}$ of dry biopolymer was used to treat $1 \mathrm{~cm}^{2}$ and was removed as the dry biopolymer $(<0.01 \mathrm{~mL}$ final volume). We have shown that the biopolymer is removable dry without secondary washing. In another experiment (not shown), the charged biopolymer solution was compared with the use of a commercial 

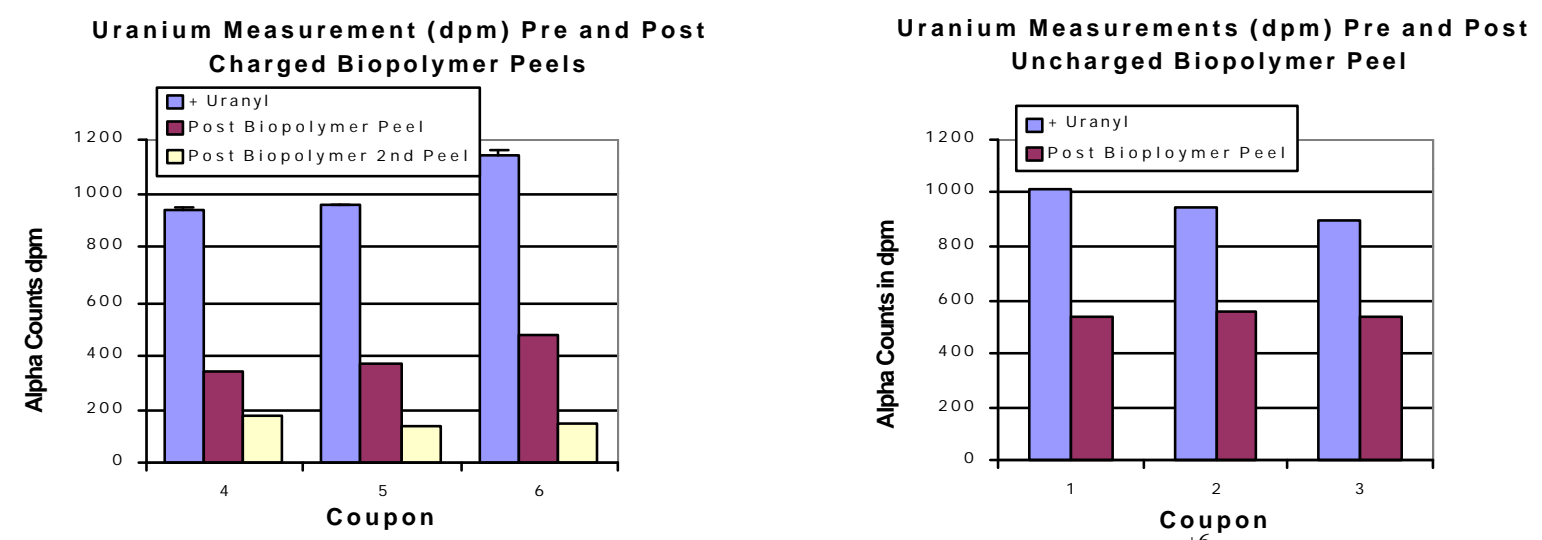

Figure 3. The charged RPS biopolymer fraction removed $>80 \%$ of surface-bound $\mathrm{U}^{+6}$, as assessed by both radioactive alpha disintegration and inductively coupled plasma spectroscopy.

strippable coating ("StripCoat", Sanchem, Inc.). In these tests, StripCoat removed $\sim 30 \%$ of the contamination, while the biopolymer solution removed $45 \%$ of surface-bound uranium after $24 \mathrm{~h}$. In Florida International University's field tests on actual surfaces, StripCoat removed about $80 \%$ of the contamination (Madaris, 1999). The discrepancy between the absolute removal by StripCoat may relate to the amount of loose contamination on the actual SRS surfaces and to the nonoptimized nature of the biopolymer tests. Nevertheless, in direct comparison, our biopolymer is clearly competitive with StripCoat and potentially more effective than other non-biological strategies.

These experiments strongly support the hypothesis that physical removal can be enhanced by sorption. StripCoat and other commercial coating do not sorb the metals; they are presumed to have a purely physical method of removal. The charged biopolymer was known to have higher metal capacities, and thus would have the greatest enhancing effect compared to the uncharged biopolymer. Other possible explanations for the extent of binding that require further study include purely chemical effects -- such as aqueous oxidation of the steel surface or low $\mathrm{pH}$.

\section{PLANNED ACTIVITIES}

This system has not been directly applied at a contaminated site. Currently, we are testing a layered application of biopolymer and StripCoat for easier removal. We are planning tests with actual contaminated metal later this year during a no cost extension until 4/02. We are preparing several manuscripts and presentations. We have submitted an EMSP renewal to address additional critical questions and hypotheses which have arisen during this project: 1] how to enhance the impact of biosorption in addition to the physical removal of contaminants by the biopolymer? 2] What are the critical characteristics and treatments of biopolymer that make it a good metal chelator and coating? and 3] How can biopolymer production be stimulated in cyanobacteria?

\section{INFORMATION ACCESS}

"Green Biopolymers for Decontamination" - poster presentation at "Workshop on integration of end user needs with research projects for EMSP: Focus on Deactivation and Decommissioning" at Savannah River Site on Nov. 17-18, 1998.

Presentation, "Green Biopolymer for Decon of Contaminated Surfaces" delivered at Decontamination, Demolition and Restoration (DD\&R) Topical Meeting on Site Restoration of Government and Commercial Facilities, Sep. 12-16, 1999, Knoxville, TN.

Davison, BH, and T Kuritz, 2000 "Peeling Off Contamination" Initiatives in Environmental Technology Investment 7 (Fall): 12. 